\title{
Cost Containment in Healthcare
}

Cost containment in healthcare can be defined as the conscious activities and policies, processes and norms adopted for reducing the cost without compromising the outcome and quality of care. In today's healthcare scenario, healthcare expenditure even in developed countries has increased by $5-7 \%$ of GDP. Per capita cost of healthcare has similarly increased. Elderly, representing $11 \%$, use about $30 \%$ of healthcare costs. In our country, out of pocket expenditure by the clientele is maximum. In this dynamic environment it is imperative that cost containment is one of the major drivers for healthcare industry to grow further.

Cost containment is the business practice of maintaining expense levels to prevent unnecessary spending or thoughtfully reducing expenses to improve profitability without long-term damage to the company. Cutting expenses in ways that decrease quality or reduce marketing efforts to bring in new business can lead to a company's demise and are not examples of a cost-containment strategy.

Healthcare ecosystems involve the balance between the demographic and epidemiological developments, the progress in biomedical technology, the strong efforts to control supply, demand, and high medical costs, as well as the continuous exchange of ideas and experiences, ensuring quality health care and reaching out to the huge underprivileged ones. Actual trends, regardless of the organizational and financial structure of the healthcare system, point to cost-containment policies in the public or private health sector. The containment of public health expenditures is of utmost importance since their continuous and uncontrolled increase deprives other social sectors of public funds. In the private sector also, this cannot be overlooked because of inflationary tendencies in the health services cost, the burden falls primarily on the routine budget with a direct and indirect impact on the economy in general, leading to inequalities in the provision of health care and the access to healthcare services.

The doctor is the final health services administrator, exercising an influence on the diagnosis and treatment process. Hence, it becomes morally binding on him to strike the ethical as well as social balance to deliver effective health care. Various operational research tools such as cost-benefit analysis, program evaluation and review technique, break-even analysis, operational audits, are some of the methods to monitor cost containment. Thus cost-containment policies in the health sector do not mean cuts in health expenses, but rational and efficient allocation of scarce resources.

The problem is known as "cost disease" - the rapidly escalating costs of basic human services like health care, housing, education, construction, and infrastructure. Cost-containment measures in healthcare aim to prevent unnecessary tests, treatments, medical devices, or surgical procedures. Uncompensated care is overall measures of services provided for which no payments were received from the patient, client, or third-party payer.

Insurance sector is growing very rapidly and organizations, including government, are promoting this aggressively. The managed care, the insurance company, makes all-out effort to decrease healthcare costs and coordinates medical services. The two main types are: preferred provider organizations (PPOs) and health maintenance organizations (HMOs). PPOs contain cost by negotiating discounts for services with hospitals and physicians as a condition for being included in the organization. Receiving a discount allows the PPOs to reduce health insurance premiums and healthcare expenditures. In turn, the patient receives a greater percentage of cost covered by the insurance company if they stay with providers that are in the PPOs. The patient may choose a provider that is not in the PPOs, but will pay a higher amount out of his amount. HMOs provide healthcare services to members for a fixed yearly fee per member. Providing too much or too costly care, could cause them to lose money. Preventative care is encouraged with this type of structure, in an attempt to avoid more costly corrective care.

Efficiency measures, also known as cost-control measures, serve many important purposes, like measure of performance of one unit relative to other units of the same organization and as a measure of performance between similar organizations. It also facilitates performance auditing and acts as a tool for analyzing and controlling the cost of inputs, as well as an evaluation criterion for accrediting agencies and regulatory bodies.

The development of efficiency measures depends on the effectiveness of an organization's financial system. The process of determining efficiency measures needs information from different parts of the financial system such as financial and cost accounting, budgeting, and auditing. Once efficiency measures are developed, these serve as important ingredients in managerial decision-making in budgeting and may also serve as important benchmarks in both financial and performance auditing. The feedback mechanism between budgeting and auditing follows the accounting cycle.

Reducing patient length of stay has been one of the main targets for clinical cost containment in hospitals. Other method by which hospitals can contain costs is by implementing electronic alerting systems that notify clinicians when they've ordered tests or antibiotics with less expensive alternatives.

Some of the biggest challenges facing providers interested in implementing cost-containment strategies include capital investment requirement, which is a huge determining factor for healthcare organizations growth. Simultaneously emerging trends like data analytics and electronic health records are stepping in a big way. Artificial intelligence has also revolutionized healthcare delivery. Time is not far when various diagnostic services may be controlled under the domain of artificial intelligence thus leading to optimization of healthcare services. It is imperative that measures should be put in place to reduce litigations and fostering a culture of finding competent physician champions, administrative leaders or a clinical optimization officer to spearhead cost-containment efforts.

The scenario pertaining to healthcare remains grim in India in spite of much efforts. The doctor-patient ratio in the country is much lower than that advocated by the WHO. However, with the advent of technology and its spread to the healthcare sector, this gap is decreasing and time will bridge the gap with continuous efforts from all sides. Moreover, the change due to introduction of universal healthcare umbrella scheme of Ayushman Bharat is awaited despite the several odds facing its implementation and success. 
There are many potential dangers of conflating cost containment and care coordination. It causes the merits of care coordination to be judged on the basis of savings, diminishing the importance of coordinated care itself as a worthy goal that can enhance patient experiences and improve outcomes even if it does not reduce utilization or produce net savings.

It could also prevent a meaningful science of waste reduction from emerging in health care. An overemphasis on care coordination as a cost-cutting strategy could divert attention and resources away from the development of approaches that eliminate the provision of low-value services more effectively. Innovation in this area is sorely needed but undercapitalized relative to the burgeoning industry of health analytics companies promising big returns from patient engagement and seamless care.

The existing evidence shows that the effectiveness of cost-containment policies varies greatly between policies, underlining the need for evidence. Future policy evaluations should focus on the effectiveness of cost-benefit analysis, cost-effect analysis and cost utility analysis as many evaluations do not take into account cost-shifting possibilities by providers and patients.

It is time that cost-containment strategies are augmented with evidence-based studies and this much-needed concept is realized to benefit maximum clientele.

Shakti Kumar Gupta

Medical Superintendent

All India Institute of Medical Sciences

Dr RP Centre for Ophthalmic Sciences

New Delhi, India

Editor-in-Chief, IJRFHHA

Maj Gen Sunil Kant, VSM

MG (Med), EC

Editor-in-Chief, IJRFHHA 